\title{
Damage Caused by Psyllids and Influence of Climatic Factors in Brazilian Accesses of Guava Trees Cultivated in Organic System
}

\author{
Juliana Altafin Galli1 ${ }^{*}$, Marcos Doniseti Michelotto' ${ }^{1}$, Maria Beatriz Bernardes Soares ${ }^{1}$, \\ Antônio Lucio Melo Martins' ${ }^{1}$, Ivan Herman Fischer ${ }^{2}$ \\ ${ }^{1}$ Apta North Centre, Pindorama, Brazil \\ ${ }^{2}$ Apta Midwest, Bauru, Brazil \\ Email: “julianagalli@apta.sp.gov.br, michelotto@apta.sp.gov.br, beatriz@apta.sp.gov.br, \\ Imartins@apta.sp.gov.br, ihfische@apta.sp.gov.br
}

Received 22 January 2014; revised 6 April 2014; accepted 18 April 2014

Copyright (C) 2014 by authors and Scientific Research Publishing Inc.

This work is licensed under the Creative Commons Attribution International License (CC BY). http://creativecommons.org/licenses/by/4.0/

(c) (i) Open Access

\begin{abstract}
The psyllid (Triozoida sp.) is the primary pest of guava tree in the Central Northern region of São Paulo State, Brazil. The variation of climatic factors may influence directly or indirectly the behavior of agricultural pests. The present work had objectives to evaluate the damages caused by psyllid in accesses of guava trees in order to identify materials with potential resistance to pest and the possible correlations of the damage with the meteorological factors, in an orchard of guava conducted in an organic system. Eighty-five guava accesses were evaluated. Each one was analyzed at random 10 leaves containing the symptom of the psyllid attack, through visual range of notes. The averages of the notes were compared by Scott-Knott test at 5\% probability. The 25 accesses with the highest average damage of psyllid had the data submitted to correlation (Pearson), with the minimum and maximum temperature $\left({ }^{\circ} \mathrm{C}\right)$, precipitation $(\mathrm{mm})$ and relative humidity (\%). Guava accesses used commercially were the most susceptible to the attack of psyllid, compared to the selections, with an emphasis to access "L4P14", "L7P28" and "L8P32B", which were the least attacked. The study of correlation between psyllid damages and meteorological factors, must be used the medium temperature, relative humidity and rainfall accumulated in the period of 14 days before the evaluations. There is a positive correlation between minimum temperasture, precipitation and relative humidity with the damage of psyllids in leaves of guava.
\end{abstract}

\section{Keywords}

Triozoida sp., Psidium guajava, Varietal Resistance, Temperature, Precipitation, Relative Humidity

\footnotetext{
${ }^{*}$ Corresponding author.
}

How to cite this paper: Galli, J.A., et al. (2014) Damage Caused by Psyllids and Influence of Climatic Factors in Brazilian Accesses of Guava Trees Cultivated in Organic System. American Journal of Plant Sciences, 5, 1432-1440. 


\section{Introduction}

The guava tree is one among many farmed species that has high genetic diversity. However, there are few varieties used commercially, both for industry and for consumption "in natura".

The production of guava in the northern region of the São Paulo State corresponds to $70 \%$ of state production [1]. Currently, in order to achieve the demand of guava fruit throughout the year, producers have irrigated the area and made constant pruning, which has caused an increase and also regularity in production. It is assumed that this change of cultural practices has led to an increase in regional population of psyllids (Triozoidalimbata) (Enderlein, 1918) (Hemiptera: Triozoidae), which today are cited as key pest of culture [2].

The characteristic symptom of guava psyllid attack is the winding from the edges of the leaves, where there are colonies of nymphs. Initially, these edges are of a yellowish (chlorotic areas) or reddish coloration. Later, with necrotic appearance, fall leaves may occur, causing some reduction in leaf area and, consequently, compromise in production [3].

Upon the damage caused by $T$. limbata, the occurrence of this insect throughout the year, the limited number of insecticides registered and the factors that make it difficult to contact insecticide with the nymphs, as they are protected inside the coiled leaf, studies are required to subsidize the development of management strategies for this pest on guava orchards [4].

Unlike the conventional system of fruit production, organic orchards are established techniques of certification programs to suppress the use of synthetic agricultural inputs. In this type of system, the production of guava in Brazil is still very little explored, by requiring greater care, mainly in plant disease area [5].

The variation of climate components can directly or indirectly influence the behavior of agricultural pests. These elements act directly on mortality and on performance of pests through changes in oviposition, feeding, growth and migration [6]. Among the climate elements, temperature, relative humidity and precipitation are the main factors related to population dynamics of agricultural pests [7].

By the above, the present work had objectives to evaluate the damage caused by psyllid in guava accesses cultivated in organic system, in order to identify possible materials with resistance to insect, and study the possible correlations between this pest and the meteorological factors temperature, precipitation and relative humidity.

\section{Material and Methods}

The experiment was developed in Apta North Centre in Pindorama, São Paulo State, Brazil $\left(21^{\circ} 11^{\prime} 9\right.$ "S and $48^{\circ} 54^{\prime} 25^{\prime \prime} \mathrm{W}$ ), with annual average temperature of $22.8^{\circ} \mathrm{C}$, annual average precipitation of $1390.3 \mathrm{~mm}$ and average annual relative humidity of $71.6 \%$. According to the Köppen classification, the climate fits the type Aw, defined as tropical humid, with rainy season in summer and dry in winter. Were used guava plants belonging to Active Germplasm Bank, containing 85 accesses, with 15 years old, being three plants/access, grown in an organic system, spaced $6 \times 5 \mathrm{~m}$. Upon the result of the analysis of soil, took place an application on surface of 1.5 ton/ha of dolomitic limestone in total area and $20 \mathrm{~L}$ of filter cake, around the trunk, in each plant. The control of spontaneous plants was accomplished by ecological reaping machine and hoeing. In July 2012, the plants suffered a drastic pruning, so that the main trunk has a height of 1.20 meters from the ground. On the occasion of this pruning, plants were with new shoots in the evaluation period. For the study with psyllid, on each access were analyzed at random 10 leaves containing the symptom of psyllid attack (edges winding), totaling 850 leaves per assessment. For each leaf sampled was assigned a note from 1 to 4 [2], who assigns a variable percentage of damage, being note 1 of 0 to $25 \%$ damage; 2 , of $26 \%$ to $50 \% ; 3,51 \%$ to $75 \%$ and note $4,76 \%$ to $100 \%$ of damage to the leaf edge. The evaluations were conducted in 10/1/12, 10/15/12, 10/29/12, 11/12/12, 11/26/12, $1 / 14 / 13,1 / 28 / 13$ and $2 / 18 / 13$. The evaluations that presented the highest notes of visible symptoms of attack were used for the analysis of the psyllid's damage (average of five final evaluations of each access), for best interpretation of the data. The averages of each access in each assessment, and the averages of the five latest evaluations, were compared by Scott-Knott test at $5 \%$ probability. For statistical analysis, the data were transformed into $\sqrt{\mathrm{x}}$, and in the table are the original values. The average data relating to psyllid damage in 25 accesses more attacked by the insect have been recorded in each evaluation and submitted to correlation (Pearson), with the minimum temperature $\left({ }^{\circ} \mathrm{C}\right)$, the maximum temperature $\left({ }^{\circ} \mathrm{C}\right)$, relative humidity (obtained at 07:00 a.m. and 01:00 p.m. hours) and precipitation ( $\mathrm{mm}$ ). The meteorological data were obtained from the meteorological station of Apta North Centre, located about $300 \mathrm{~m}$ from the experiment. In correlations, psyllid data were corre- 
lated with meteorological factors recorded the day before the evaluations, with averages recorded during periods of seven and 14 days prior evaluations, in order to determine which one is the length of time that must be used in the correlations. For precipitation, we used the value of evening, and the accumulated values at seven and 14 days prior to assessments.

\section{Results}

It was observed an increase in the severity of insect attack from the third assessment, as shown in Figure 1.

The statistical analysis of the notes obtained in evaluations is in Table 1. The average of the last five evaluations allowed the separation of accesses into four groups, with the ones used commercially, as Rich and Paluma, the most attacked by psyllid. The L4P14, L7P28 and L8P32B accesses showed few symptoms of attack and may indicate any resistance to the insect.

The correlations between the average data relating to psyllid damage in 25 accesses more attacked with the minimum temperature $\left({ }^{\circ} \mathrm{C}\right)$, the maximum temperature $\left({ }^{\circ} \mathrm{C}\right)$ and relative humidity $(\%)$, using the average of 14 days, and for precipitation $(\mathrm{mm})$ accumulated in 14 days prior the evaluations are in Figure 2, Figure 3 and Figure 4. It was observed an increase in the severity of psyllid attack from the third assessment, probably due to the increase of new shoots.

The damage of psyllid showed positive correlation only with the relative humidity recorded at 1:00 p.m. among the meteorological factors when taken on the eve of the evaluations (Table 2). However, when one takes into account the average temperature of seven days before the assessments, there is a positive correlation of psyllid damage with minimum temperature, demonstrating that there is an increase in the damage caused by the insect with the increase of the minimum temperature. The average of temperature of 14 days prior assessments and the rainfall during this period, it appears that, in addition to the correlation with the minimum temperature, there is a significant positive correlation also with precipitation and relative humidity registered at 1:00 p.m., demonstrating that there is an increase in the damage caused by the pest with increasing precipitation.

\section{Discussion}

The increased severity of insect attack from the third assessment (Figure 1) is due to the greater number of shoots, once the psyllid posture is held over the pointers and young leaves of the guava tree [3]. Several authors have verified population peaks of psyllid in the months following the pruning [2] [8]-[12].

Colombi \& Galli [2], assessing the population fluctuation and the evolution of damage of T. limbata in guava orchards subjected to minimal use of pesticides, have noted a surge in damage, which continued increasing until September, and that this increase was due to the pruning accomplished in June, which induced the sprouting and emergence of new leaves, providing favorable conditions for the development of psyllid. The authors also con-

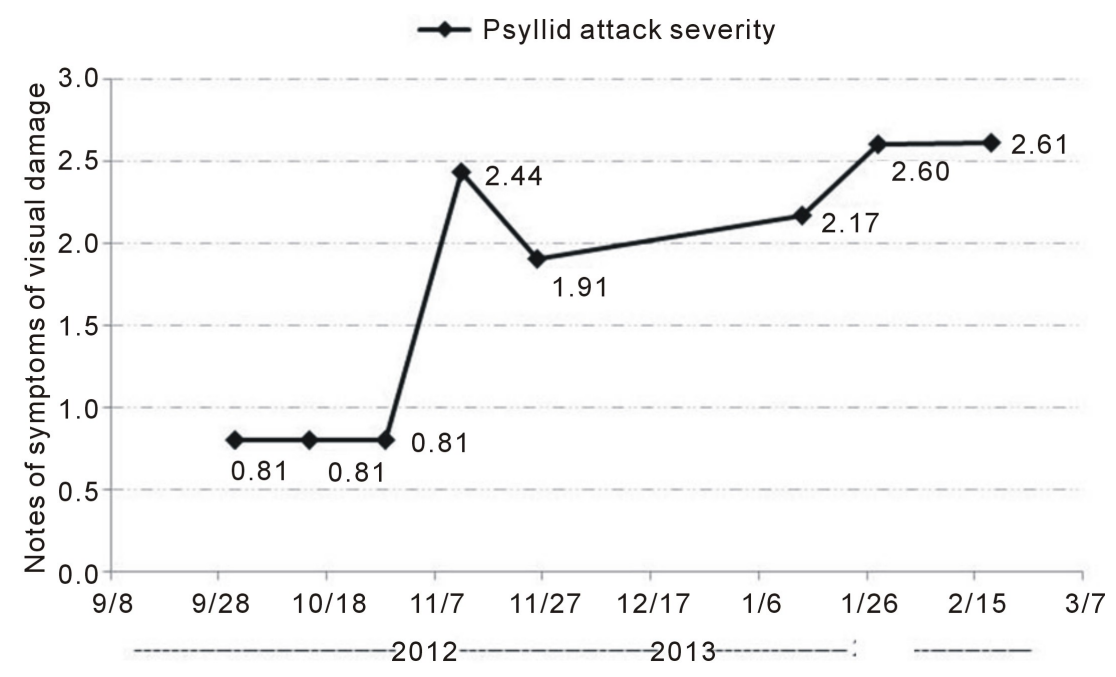

Figure 1. Average rating of visual symptoms of psyllid attack on 85 access. Pindorama/SP/Brazil, 2012/2013. 
Table 1. Notes of visual symptoms of psyllid (Triozoida sp.) attack, in guava access, on different evaluations. Pindorama/SP /Brazil, 2012/2013.

\begin{tabular}{|c|c|c|c|c|c|c|c|c|c|c|c|c|c|}
\hline \multirow{3}{*}{ Access } & \multirow{3}{*}{ Origin } & \multicolumn{10}{|c|}{ Evaluation times } & \multirow{2}{*}{\multicolumn{2}{|c|}{$\begin{array}{c}\text { Average of } 5 \\
\text { evaluations }\end{array}$}} \\
\hline & & \multicolumn{2}{|l|}{$11 / 12 / 12$} & \multicolumn{2}{|c|}{$11 / 26 / 12$} & \multicolumn{2}{|c|}{$01 / 14 / 13$} & \multicolumn{2}{|c|}{$01 / 28 / 13$} & \multicolumn{2}{|c|}{$02 / 18 / 13$} & & \\
\hline & & \multicolumn{12}{|c|}{ 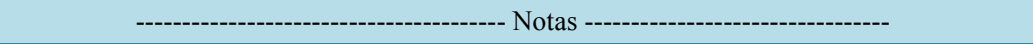 } \\
\hline EEFT1 (Almas) & Monte Alto & 3.7 & $\mathrm{a}$ & 3.7 & $\mathrm{a}$ & 4.0 & $\mathrm{a}$ & 4.0 & $\mathrm{a}$ & 4.0 & $\mathrm{a}$ & 3.9 & a \\
\hline RubySupreme2 & Monte Alto & 3.2 & $\mathrm{a}$ & 3.6 & $\mathrm{a}$ & 4.0 & $\mathrm{a}$ & 4.0 & $\mathrm{a}$ & 4.0 & $\mathrm{a}$ & 3.8 & $\mathrm{a}$ \\
\hline RubySupreme3 & Monte Alto & 3.5 & $\mathrm{a}$ & 2.4 & $\mathrm{~b}$ & 3.9 & $\mathrm{a}$ & 4.0 & $\mathrm{a}$ & 4.0 & $\mathrm{a}$ & 3.6 & $\mathrm{a}$ \\
\hline Patillo-Ba. & Jundiaí & 3.2 & $\mathrm{a}$ & 3.7 & $\mathrm{a}$ & 3.4 & $\mathrm{a}$ & 3.9 & $\mathrm{a}$ & 3.3 & $\mathrm{a}$ & 3.5 & $\mathrm{a}$ \\
\hline RedSelection & Monte Alto & 3.6 & $\mathrm{a}$ & 3.3 & $\mathrm{a}$ & 3.3 & $\mathrm{a}$ & 3.0 & $\mathrm{~b}$ & 4.0 & $\mathrm{a}$ & 3.4 & $\mathrm{a}$ \\
\hline M. Alto Comum 1 & Monte Alto & 3.3 & $\mathrm{a}$ & 3.0 & $\mathrm{a}$ & 3.4 & $\mathrm{a}$ & 3.7 & $\mathrm{a}$ & 3.4 & $\mathrm{a}$ & 3.4 & $\mathrm{a}$ \\
\hline Kioshi2 & Monte Alto & 2.8 & $\mathrm{~b}$ & 2.2 & $\mathrm{c}$ & 3.5 & $\mathrm{a}$ & 4.0 & $\mathrm{a}$ & 4.0 & $\mathrm{a}$ & 3.3 & $\mathrm{a}$ \\
\hline Rica-J-2 & Jaboticabal & 3.9 & $\mathrm{a}$ & 1.6 & $\mathrm{~d}$ & 3.1 & $\mathrm{a}$ & 4.0 & $\mathrm{a}$ & 3.8 & $\mathrm{a}$ & 3.3 & $\mathrm{a}$ \\
\hline Webber Supreme & Jaboticabal & 3.8 & $\mathrm{a}$ & 1.9 & $\mathrm{c}$ & 1.5 & $\mathrm{c}$ & 2.9 & $\mathrm{~b}$ & 2.6 & $\mathrm{~b}$ & 3.1 & $\mathrm{a}$ \\
\hline Paluma & Jaboticabal & 2.8 & $\mathrm{~b}$ & 2.1 & $\mathrm{c}$ & 2.6 & $\mathrm{~b}$ & 4.0 & $\mathrm{a}$ & 3.8 & $\mathrm{a}$ & 3.1 & $\mathrm{a}$ \\
\hline EEFT 2 (Almas) & Monte Alto & 3.7 & $\mathrm{a}$ & 1.6 & $\mathrm{~d}$ & 3.3 & $\mathrm{a}$ & 3.4 & $\mathrm{a}$ & 3.3 & $\mathrm{a}$ & 3.1 & $\mathrm{a}$ \\
\hline Creme arredon. & Jaboticabal & 3.5 & $\mathrm{a}$ & 2.1 & $\mathrm{c}$ & 3.1 & $\mathrm{a}$ & 3.7 & $\mathrm{a}$ & 2.8 & $\mathrm{~b}$ & 3.0 & $\mathrm{a}$ \\
\hline FAO_-Unesp & Jaboticabal & 2.9 & $\mathrm{~b}$ & 1.7 & $\mathrm{c}$ & 3.5 & $\mathrm{a}$ & 3.3 & $\mathrm{a}$ & 3.6 & $\mathrm{a}$ & 3.0 & $\mathrm{a}$ \\
\hline IAC-4_-Unesp & Jaboticabal & 2.8 & $\mathrm{~b}$ & 1.8 & $\mathrm{c}$ & 2.8 & $\mathrm{~b}$ & 4.0 & $\mathrm{a}$ & 3.6 & $\mathrm{a}$ & 3.0 & $\mathrm{a}$ \\
\hline Saito & Valinhos & 2.6 & $\mathrm{~b}$ & 1.9 & $\mathrm{c}$ & 3.3 & $\mathrm{a}$ & 4.0 & $\mathrm{a}$ & 3.0 & $\mathrm{~b}$ & 3.0 & $\mathrm{a}$ \\
\hline Kioshi1 & Monte Alto & 1.8 & $\mathrm{c}$ & 2.2 & $\mathrm{c}$ & 3.3 & $\mathrm{a}$ & 4.0 & $\mathrm{a}$ & 3.4 & $\mathrm{a}$ & 2.9 & $\mathrm{a}$ \\
\hline M.Alto-Branca & Jundiaí & 2.5 & $\mathrm{~b}$ & 2.5 & $\mathrm{~b}$ & 2.6 & $\mathrm{~b}$ & 4.0 & $\mathrm{a}$ & 3.1 & $\mathrm{~b}$ & 2.9 & $\mathrm{a}$ \\
\hline Kioshi3 & Monte Alto & 3.2 & $\mathrm{a}$ & 2.9 & $\mathrm{a}$ & 2.8 & $\mathrm{~b}$ & 2.5 & $\mathrm{c}$ & 3.1 & $\mathrm{~b}$ & 2.9 & $\mathrm{a}$ \\
\hline EEF-3 & Jaboticabal & 3.3 & $\mathrm{a}$ & 1.9 & $\mathrm{c}$ & 2.6 & $\mathrm{~b}$ & 3.4 & $\mathrm{a}$ & 3.2 & $\mathrm{~b}$ & 2.9 & $\mathrm{a}$ \\
\hline EEFT 3 & Monte Alto & 3.5 & $\mathrm{a}$ & 3.6 & $\mathrm{a}$ & 2.2 & $\mathrm{~b}$ & 3.1 & $\mathrm{~b}$ & 2.0 & $\mathrm{c}$ & 2.9 & $\mathrm{a}$ \\
\hline Verm. Perfum. & Jundiaí & 2.9 & $\mathrm{~b}$ & 1.7 & $\mathrm{c}$ & 3.0 & $\mathrm{a}$ & 2.6 & $\mathrm{c}$ & 4.0 & $\mathrm{a}$ & 2.8 & $\mathrm{a}$ \\
\hline Ogawa $\times$ Kumagai & Promissão & 2.8 & $\mathrm{~b}$ & 2.3 & $\mathrm{~b}$ & 3.4 & $\mathrm{a}$ & 2.4 & $\mathrm{c}$ & 3.2 & $\mathrm{~b}$ & 2.8 & $\mathrm{a}$ \\
\hline EEFT 4 & Monte Alto & 3.5 & $\mathrm{a}$ & 3.2 & $\mathrm{a}$ & 2.5 & $\mathrm{~b}$ & 2.3 & $\mathrm{c}$ & 2.4 & $\mathrm{c}$ & 2.8 & $\mathrm{a}$ \\
\hline RS EEFT CAB.A. & Jundiaí & 3.0 & $\mathrm{~b}$ & 2.3 & $\mathrm{~b}$ & 2.2 & $\mathrm{c}$ & 3.0 & $\mathrm{~b}$ & 3.4 & $\mathrm{a}$ & 2.8 & $\mathrm{a}$ \\
\hline L8P30 & Campinas & 3.7 & $\mathrm{a}$ & 2.0 & $\mathrm{c}$ & 2.1 & $\mathrm{c}$ & 2.9 & $\mathrm{~b}$ & 3.1 & $\mathrm{~b}$ & 2.8 & $\mathrm{a}$ \\
\hline Taquar. comum & Monte Alto & 2.5 & $\mathrm{~b}$ & 2.4 & $\mathrm{~b}$ & 1.9 & $\mathrm{c}$ & 3.3 & $\mathrm{a}$ & 3.5 & $\mathrm{a}$ & 2.7 & $\mathrm{~b}$ \\
\hline FAOEEFTCABA & Jundiaí & 2.3 & $\mathrm{c}$ & 1.9 & $\mathrm{c}$ & 2.6 & $\mathrm{~b}$ & 3.3 & $\mathrm{a}$ & 3.5 & $\mathrm{a}$ & 2.7 & $\mathrm{~b}$ \\
\hline L1P3 & Campinas & 2.5 & $\mathrm{~b}$ & 1.9 & $\mathrm{c}$ & 2.5 & $\mathrm{~b}$ & 3.4 & $\mathrm{a}$ & 3.2 & $\mathrm{~b}$ & 2.7 & $\mathrm{~b}$ \\
\hline Ogawa3 & Mogi Cruzes & 3.2 & $\mathrm{a}$ & 2.5 & $\mathrm{~b}$ & 1.7 & $\mathrm{c}$ & 3.1 & $\mathrm{~b}$ & 3.0 & $\mathrm{~b}$ & 2.7 & $\mathrm{~b}$ \\
\hline Mirtácea & Campinas & 2.1 & $\mathrm{c}$ & 1.3 & $\mathrm{~d}$ & 2.3 & $\mathrm{~b}$ & 3.8 & $\mathrm{a}$ & 3.9 & $\mathrm{a}$ & 2.7 & $\mathrm{~b}$ \\
\hline L1P2 & Campinas & 2.8 & $\mathrm{~b}$ & 1.9 & $\mathrm{c}$ & 2.6 & $\mathrm{~b}$ & 2.8 & $\mathrm{~b}$ & 3.1 & $\mathrm{~b}$ & 2.6 & $\mathrm{~b}$ \\
\hline L4P17 & Campinas & 2.9 & $\mathrm{~b}$ & 1.4 & $\mathrm{~d}$ & 2.7 & $\mathrm{~b}$ & 2.7 & $\mathrm{~b}$ & 3.2 & $\mathrm{~b}$ & 2.6 & $\mathrm{~b}$ \\
\hline Supreme & Monte Alto & 3.0 & $\mathrm{~b}$ & 2.2 & $\mathrm{c}$ & 2.4 & $\mathrm{~b}$ & 2.5 & $\mathrm{c}$ & 2.8 & $\mathrm{~b}$ & 2.6 & $\mathrm{~b}$ \\
\hline L3P8 & Campinas & 2.7 & $\mathrm{~b}$ & 1.9 & $\mathrm{c}$ & 1.4 & $\mathrm{~d}$ & 3.1 & $\mathrm{~b}$ & 3.7 & $\mathrm{a}$ & 2.6 & $\mathrm{~b}$ \\
\hline L3P7 & Campinas & 2.9 & $\mathrm{~b}$ & 2.1 & $\mathrm{c}$ & 2.6 & $\mathrm{~b}$ & 2.5 & $\mathrm{c}$ & 2.7 & $\mathrm{~b}$ & 2.6 & $\mathrm{~b}$ \\
\hline L8P32A & Campinas & 2.8 & $\mathrm{~b}$ & 2.1 & $\mathrm{c}$ & 1.9 & $\mathrm{c}$ & 3.5 & $\mathrm{a}$ & 2.5 & $\mathrm{~b}$ & 2.6 & $\mathrm{~b}$ \\
\hline Prop. TataoOgawa & Mogi Cruzes & 2.4 & $\mathrm{c}$ & 1.4 & $\mathrm{~d}$ & 1.7 & $\mathrm{c}$ & 3.3 & $\mathrm{a}$ & 3.8 & $\mathrm{a}$ & 2.5 & $\mathrm{~b}$ \\
\hline L5P20 & Campinas & 3.4 & $\mathrm{a}$ & 2.1 & $\mathrm{c}$ & 1.8 & $\mathrm{c}$ & 2.5 & $\mathrm{c}$ & 2.6 & $\mathrm{~b}$ & 2.5 & $\mathrm{~b}$ \\
\hline Austr.branca & Jundiaí & 2.6 & $\mathrm{~b}$ & 2.2 & $\mathrm{c}$ & 2.6 & $\mathrm{~b}$ & 2.7 & $\mathrm{~b}$ & 2.1 & $\mathrm{c}$ & 2.4 & $\mathrm{~b}$ \\
\hline Patillo & Jaboticabal & 2.0 & $\mathrm{c}$ & 1.1 & $\mathrm{~d}$ & 2.6 & $\mathrm{~b}$ & 3.0 & $\mathrm{~b}$ & 3.5 & $\mathrm{a}$ & 2.4 & $\mathrm{~b}$ \\
\hline Monte Alto & Jundiaí & 2.4 & $\mathrm{c}$ & 1.8 & $\mathrm{c}$ & 1.6 & $\mathrm{c}$ & 2.8 & $\mathrm{~b}$ & 3.5 & $\mathrm{a}$ & 2.4 & $\mathrm{~b}$ \\
\hline Ogawa1 & Mogi Cruzes & 1.9 & $\mathrm{c}$ & 1.3 & $\mathrm{~d}$ & 2.6 & $\mathrm{~b}$ & 3.5 & $\mathrm{a}$ & 2.8 & $\mathrm{~b}$ & 2.4 & $\mathrm{~b}$ \\
\hline L3P11 & Campinas & 2.6 & $\mathrm{~b}$ & 2.0 & $\mathrm{c}$ & 2.0 & $\mathrm{c}$ & 2.4 & $\mathrm{c}$ & 2.9 & $\mathrm{~b}$ & 2.4 & $\mathrm{~b}$ \\
\hline L3P12 & Campinas & 2.7 & $\mathrm{~b}$ & 1.8 & $\mathrm{c}$ & 2.8 & $\mathrm{~b}$ & 1.8 & $\mathrm{~d}$ & 2.8 & $\mathrm{~b}$ & 2.4 & $\mathrm{~b}$ \\
\hline
\end{tabular}




\section{Continued}

\begin{tabular}{|c|c|c|c|c|c|c|c|c|c|c|c|c|c|}
\hline L4P15 & Campinas & 3.1 & $\mathrm{a}$ & 2.6 & $\mathrm{~b}$ & 1.3 & $\mathrm{~d}$ & 2.3 & $\mathrm{c}$ & 2.4 & $\mathrm{c}$ & 2.3 & $\mathrm{~b}$ \\
\hline L6P22 & Campinas & 2.1 & $\mathrm{c}$ & 2.2 & $\mathrm{c}$ & 1.7 & $\mathrm{c}$ & 3.1 & $\mathrm{~b}$ & 2.3 & $\mathrm{c}$ & 2.3 & $\mathrm{~b}$ \\
\hline L2P5 & Campinas & 2.1 & $\mathrm{c}$ & 2.0 & $\mathrm{c}$ & 2.1 & $\mathrm{c}$ & 2.7 & $\mathrm{~b}$ & 2.6 & $\mathrm{~b}$ & 2.3 & $\mathrm{~b}$ \\
\hline L4P16 & Campinas & 2.2 & $\mathrm{c}$ & 2.2 & $\mathrm{c}$ & 1.7 & $\mathrm{c}$ & 3.0 & $\mathrm{~b}$ & 2.2 & $\mathrm{c}$ & 2.3 & $\mathrm{~b}$ \\
\hline Supreme BA. & Jundiaí & 2.2 & $\mathrm{c}$ & 1.6 & d & 1.8 & $\mathrm{c}$ & 2.6 & $\mathrm{c}$ & 3.0 & $\mathrm{~b}$ & 2.2 & $\mathrm{~b}$ \\
\hline $\mathrm{L} 4 \mathrm{P} 13$ & Campinas & 1.6 & d & 1.7 & $\mathrm{c}$ & 1.7 & $\mathrm{c}$ & 3.0 & $\mathrm{~b}$ & 3.2 & $\mathrm{~b}$ & 2.2 & $\mathrm{~b}$ \\
\hline L7P26 & Campinas & 1.8 & $\mathrm{c}$ & 1.7 & $\mathrm{~d}$ & 1.6 & $\mathrm{c}$ & 2.9 & $\mathrm{~b}$ & 3.1 & $\mathrm{~b}$ & 2.2 & $\mathrm{~b}$ \\
\hline Indiana & Jundiaí & 1.6 & d & 2.0 & $\mathrm{c}$ & 2.4 & $\mathrm{~b}$ & 2.8 & $\mathrm{~b}$ & 2.2 & $\mathrm{c}$ & 2.2 & $\mathrm{~b}$ \\
\hline Ver. (Shimoda) & Valinhos & 2.1 & $\mathrm{c}$ & 0.6 & $\mathrm{~d}$ & 2.1 & $\mathrm{c}$ & 2.1 & $\mathrm{c}$ & 2.8 & $\mathrm{~b}$ & 2.1 & $\mathrm{~b}$ \\
\hline Kumagai branca & Valinhos & 2.5 & $\mathrm{~b}$ & 1.6 & d & 2.2 & $\mathrm{~b}$ & 2.3 & $\mathrm{c}$ & 2.1 & $\mathrm{c}$ & 2.1 & $\mathrm{~b}$ \\
\hline Webber Supreme & Jundiaí & 2.1 & $\mathrm{c}$ & 2.7 & $\mathrm{~b}$ & 2.8 & $\mathrm{~b}$ & 3.7 & $\mathrm{a}$ & 2.2 & $\mathrm{c}$ & 2.1 & $\mathrm{~b}$ \\
\hline L6P24 & Campinas & 1.7 & $\mathrm{~d}$ & 1.6 & $\mathrm{~d}$ & 2.1 & $\mathrm{c}$ & 2.0 & $\mathrm{c}$ & 3.2 & $\mathrm{~b}$ & 2.1 & $\mathrm{~b}$ \\
\hline L2P6 & Campinas & 3.2 & $\mathrm{a}$ & 1.9 & $\mathrm{c}$ & 2.1 & $\mathrm{c}$ & 1.9 & $\mathrm{~d}$ & 1.5 & $\mathrm{~d}$ & 2.1 & $\mathrm{~b}$ \\
\hline Austr.Vermelha & Jundiaí & 2.6 & $\mathrm{~b}$ & 1.5 & $\mathrm{~d}$ & 1.4 & $\mathrm{~d}$ & 2.8 & $\mathrm{~b}$ & 2.2 & $\mathrm{c}$ & 2.1 & $\mathrm{~b}$ \\
\hline (Cingapura) & Linhares-ES & 2.0 & $\mathrm{c}$ & 1.5 & $\mathrm{~d}$ & 2.6 & $\mathrm{~b}$ & 2.7 & $\mathrm{~b}$ & 1.5 & $\mathrm{~d}$ & 2.06 & c \\
\hline Goiaba p. amarela & Valinhos & 2.1 & $\mathrm{c}$ & 1.3 & $\mathrm{~d}$ & 2.3 & $\mathrm{~b}$ & 2.9 & $\mathrm{~b}$ & 1.7 & d & 2.06 & c \\
\hline L3P9 & Campinas & 1.7 & d & 1.2 & $d$ & 2.2 & $\mathrm{c}$ & 2.4 & $\mathrm{c}$ & 2.6 & $\mathrm{~b}$ & 2.0 & c \\
\hline RubySupreme1 & Monte Alto & 2.2 & $\mathrm{c}$ & 1.8 & $\mathrm{c}$ & 1.7 & $\mathrm{c}$ & 2.8 & $\mathrm{~b}$ & 1.4 & $\mathrm{~d}$ & 2.0 & c \\
\hline L5P21 & Campinas & 1.3 & $\mathrm{~d}$ & 1.5 & $\mathrm{~d}$ & 1.3 & $\mathrm{~d}$ & 2.5 & $\mathrm{c}$ & 2.6 & $\mathrm{~b}$ & 1.8 & c \\
\hline L5P18 & Campinas & 1.9 & $\mathrm{c}$ & 1.8 & $\mathrm{c}$ & 1.8 & $\mathrm{c}$ & 1.8 & $\mathrm{~d}$ & 1.9 & $\mathrm{c}$ & 1.8 & c \\
\hline L3P10 & Campinas & 2.0 & $\mathrm{c}$ & 2.0 & $\mathrm{c}$ & 1.8 & $\mathrm{c}$ & 1.3 & $\mathrm{~d}$ & 2.1 & $\mathrm{c}$ & 1.8 & c \\
\hline L6P25 & Campinas & 2.0 & $\mathrm{c}$ & 1.2 & $\mathrm{~d}$ & 2.3 & $\mathrm{~b}$ & 1.0 & $\mathrm{~d}$ & 2.1 & $\mathrm{c}$ & 1.7 & c \\
\hline Indiana-UNESP & Jaboticabal & 1.5 & d & 1.3 & $\mathrm{~d}$ & 1.4 & $\mathrm{~d}$ & 2.3 & $\mathrm{c}$ & 1.9 & $\mathrm{c}$ & 1.7 & c \\
\hline Supreme branca & Jundiaí & 2.0 & $\mathrm{c}$ & 1.9 & $\mathrm{c}$ & 1.3 & $\mathrm{~d}$ & 1.4 & $\mathrm{~d}$ & 1.7 & d & 1.7 & c \\
\hline IAC-4-Cica & Monte Alto & 2.1 & $\mathrm{c}$ & 1.3 & $\mathrm{~d}$ & 1.4 & d & 2.2 & $\mathrm{c}$ & 1.2 & $\mathrm{~d}$ & 1.6 & c \\
\hline Goiaba branca & Unknown & 2.2 & $\mathrm{c}$ & 1.4 & d & 1.7 & $\mathrm{c}$ & 1.4 & $\mathrm{~d}$ & 1.5 & d & 1.6 & c \\
\hline Guanabara & Jundiaí & 1.8 & d & 1.0 & $\mathrm{~d}$ & 1.2 & d & 1.6 & $\mathrm{~d}$ & 2.4 & $\mathrm{c}$ & 1.6 & c \\
\hline $\mathrm{L} 2 \mathrm{P} 4$ & Campinas & 2.3 & $\mathrm{c}$ & 1.5 & $\mathrm{~d}$ & 1.2 & d & 1.7 & $\mathrm{~d}$ & 1.3 & $d$ & 1.6 & c \\
\hline Torrão de Ouro & Jundiaí & 1.3 & $d$ & 1.8 & $\mathrm{c}$ & 0.1 & $d$ & 1.8 & $\mathrm{~d}$ & 1.9 & $\mathrm{c}$ & 1.6 & c \\
\hline L5P19 & Campinas & 2.0 & $\mathrm{c}$ & 1.7 & $\mathrm{c}$ & 1.4 & $\mathrm{~d}$ & 1.3 & $\mathrm{~d}$ & 1.5 & $\mathrm{~d}$ & 1.6 & c \\
\hline Rubi Supreme & Jundiaí & 1.4 & d & 1.2 & $\mathrm{~d}$ & 1.6 & $\mathrm{c}$ & 1.6 & d & 2.0 & $\mathrm{c}$ & 1.6 & c \\
\hline Ver. Red. (Shim.) & Valinhos & 1.4 & $\mathrm{~d}$ & 1.5 & $\mathrm{~d}$ & 1.3 & $\mathrm{~d}$ & 1.4 & $\mathrm{~d}$ & 2.1 & $\mathrm{c}$ & 1.5 & c \\
\hline L6P23 & Campinas & 1.7 & $\mathrm{~d}$ & 1.0 & $\mathrm{~d}$ & 1.4 & $\mathrm{~d}$ & 1.5 & $\mathrm{~d}$ & 1.6 & $\mathrm{~d}$ & 1.4 & c \\
\hline IAC-4 & Jundiaí & 1.6 & $\mathrm{~d}$ & 1.2 & $\mathrm{~d}$ & 1.2 & $\mathrm{~d}$ & 1.4 & $\mathrm{~d}$ & 1.6 & $\mathrm{~d}$ & 1.4 & c \\
\hline $\mathrm{EEFT}-\mathrm{CA}-\mathrm{BA}$ & Jundiaí & 1.5 & $\mathrm{~d}$ & 1.6 & $\mathrm{~d}$ & 1.4 & $\mathrm{~d}$ & 1.3 & $\mathrm{~d}$ & 1.0 & $\mathrm{~d}$ & 1.4 & c \\
\hline L8P31 & Campinas & 1.6 & d & 1.6 & $\mathrm{~d}$ & 1.0 & $\mathrm{~d}$ & 0.6 & $\mathrm{e}$ & 1.9 & $\mathrm{c}$ & 1.3 & c \\
\hline Campos & Jundiaí & 1.4 & $\mathrm{~d}$ & 1.3 & $\mathrm{~d}$ & 1.2 & $\mathrm{~d}$ & 1.2 & $\mathrm{~d}$ & 1.4 & $\mathrm{~d}$ & 1.3 & c \\
\hline Tetraplóide & Jundiaí & 1.4 & $\mathrm{~d}$ & 1.2 & $\mathrm{~d}$ & 1.3 & $\mathrm{~d}$ & 1.0 & $\mathrm{~d}$ & 1.1 & $\mathrm{~d}$ & 1.2 & $\mathrm{~d}$ \\
\hline L4P14 & Campinas & 1.7 & d & 1.5 & d & 1.0 & d & 0.1 & $\mathrm{f}$ & 1.5 & $d$ & 1.2 & $\mathrm{~d}$ \\
\hline L7P28 & Campinas & 2.2 & $\mathrm{c}$ & 1.4 & $d$ & 1.0 & $d$ & 0.2 & $\mathrm{f}$ & 0.2 & $\mathrm{e}$ & 1.0 & $\mathrm{~d}$ \\
\hline L8P32B & Campinas & 1.0 & d & 1.4 & d & 0.4 & $\mathrm{e}$ & 0.4 & $\mathrm{e}$ & 0.3 & $\mathrm{e}$ & 0.7 & $\mathrm{~d}$ \\
\hline F (Blocks) & & \multicolumn{2}{|c|}{$1.0839^{\mathrm{NS}}$} & \multicolumn{2}{|c|}{$1.4468^{\mathrm{NS}}$} & \multicolumn{2}{|c|}{$1.3135^{\mathrm{NS}}$} & \multicolumn{2}{|c|}{$1.0228^{\mathrm{NS}}$} & \multicolumn{2}{|c|}{$0.6908^{\mathrm{NS}}$} & \multicolumn{2}{|c|}{$23.3399^{* *}$} \\
\hline F (Treatments) & & \multicolumn{2}{|c|}{$6.1636^{* *}$} & \multicolumn{2}{|c|}{$5.2656^{* *}$} & \multicolumn{2}{|c|}{$10.063^{* *}$} & \multicolumn{2}{|c|}{$20.684^{* *}$} & \multicolumn{2}{|c|}{$14.5337^{* *}$} & \multicolumn{2}{|c|}{$8.2977^{* *}$} \\
\hline CV (\%) & & \multicolumn{2}{|c|}{19.91} & \multicolumn{2}{|c|}{21.67} & \multicolumn{2}{|c|}{19.99} & \multicolumn{2}{|c|}{17.46} & \multicolumn{2}{|c|}{17.99} & \multicolumn{2}{|c|}{11.96} \\
\hline
\end{tabular}

cluded that there is a strong relationship between the damage and the population density of the insect.

Duarte et al. [12], studying the population dynamics of T. limbata, Costalimaita ferruginea (Fabr., 1801) (Col.: Chrysomelidae) and natural enemies in organic and conventional orchard of guava, verified that the population density of $T$. limbata remained low, but constant, with a peak in October, in the organic orchard, next month 


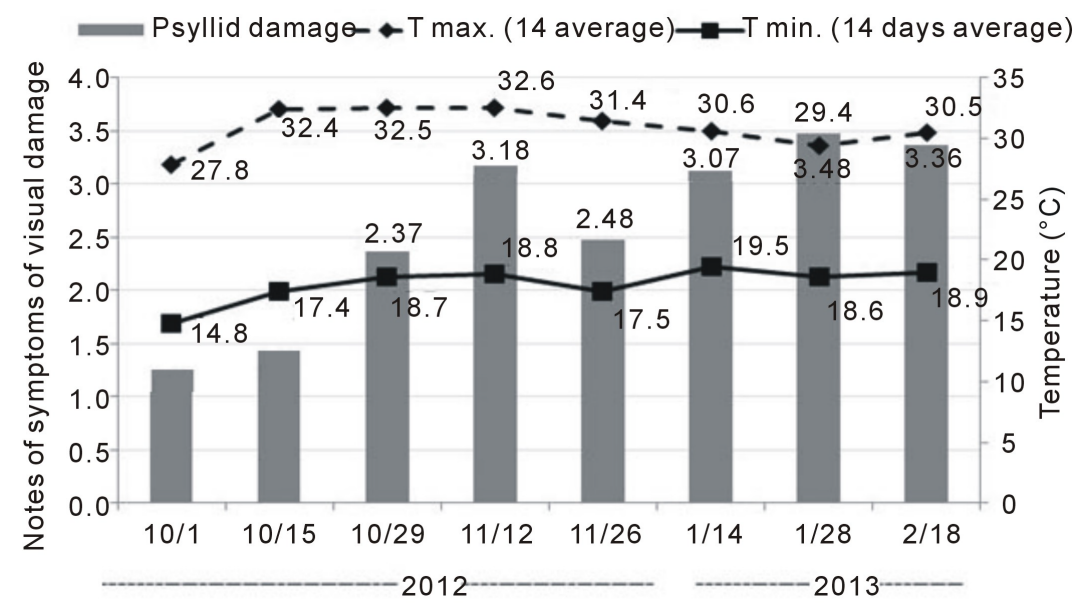

Figure 2. Average rating of visual symptoms of psyllid attack in 25 access and maximum and minimum temperatures recorded during the period. Pindorama/SP/Brazil, 2012/2013.

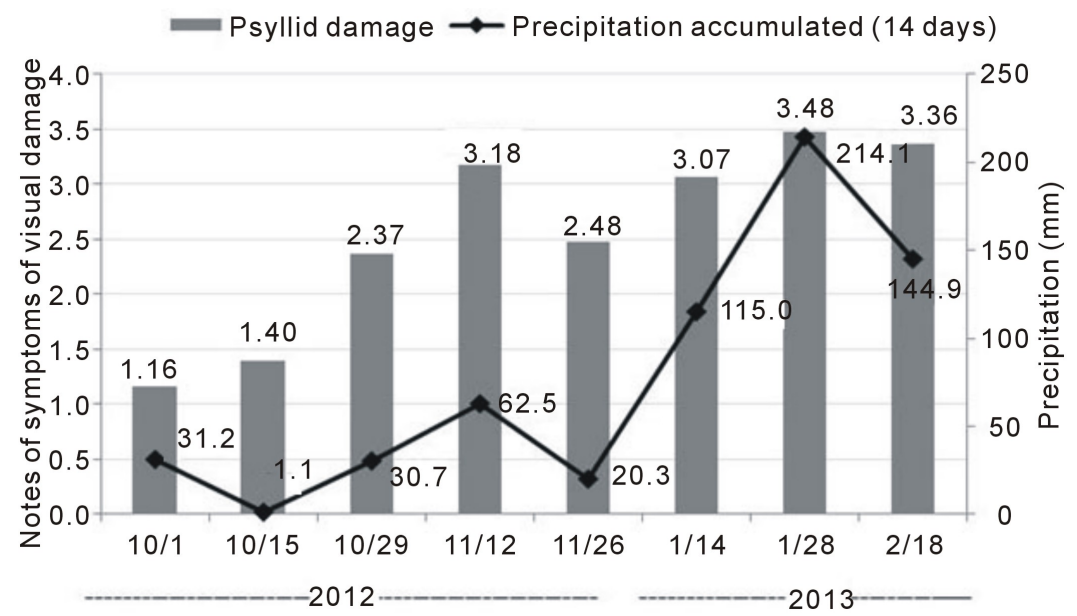

Figure 3. Average rating of visual symptoms of psyllid attack in 25 access and precipitation recorded in the period. Pindorama/SP/Brazil, 2012/2013.

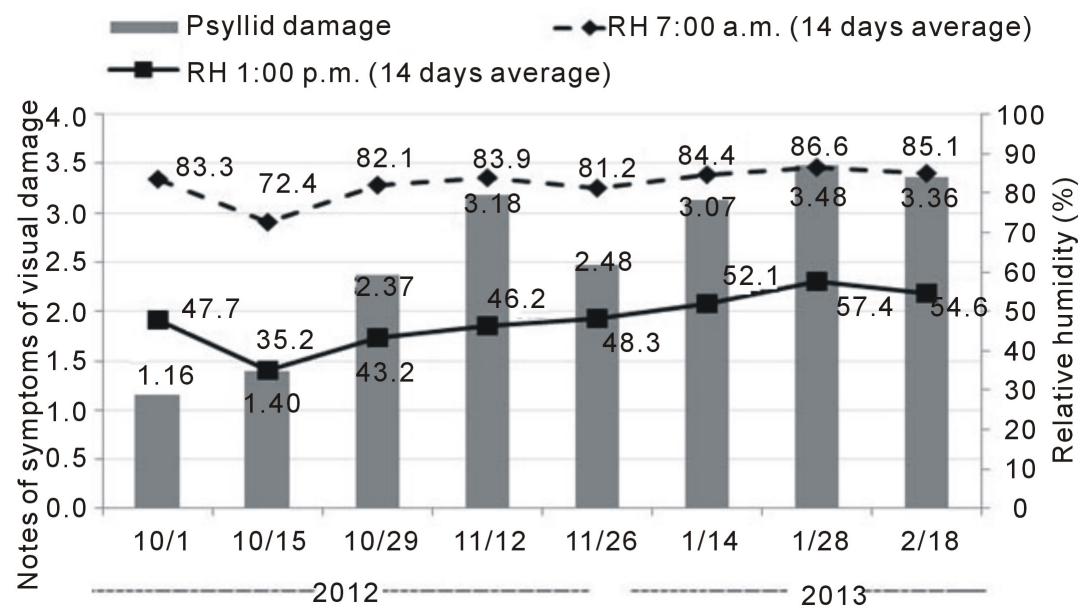

Figure 4. Average rating of visual symptoms of psyllidattack in 25 access and relative humidity recorded in the period. Pindorama/SP/Brazil, 2012/2013. 
Table 2. Coefficient of correlation between damage caused by psyllids and meteorological factors minimum temperature (TMIN), maximum temperature (TMAX), precipitation (PREC) and the relative humidity at 7:00 a.m. and 1:00 p.m. (RH) recorded on the eve of assessments, the 7 and 14 days prior assessments. Pindorama/SP/Brazil, 2012/2013.

\begin{tabular}{|c|c|c|c|c|c|}
\hline \multirow{2}{*}{ Psyllid } & \multicolumn{5}{|c|}{ Coefficient of correlation (Pearson) } \\
\hline & $\operatorname{TMIN}\left({ }^{\circ} \mathrm{C}\right)$ & $\operatorname{TMAX}\left({ }^{\circ} \mathrm{C}\right)$ & $\operatorname{PREC}(\mathrm{mm})$ & RH 7 a.m. & RH 1 p.m \\
\hline \multirow{4}{*}{ Damages } & & -------------- & gistered the da & 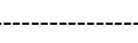 & \\
\hline & $0.6946^{\mathrm{NS}}$ & $0.0800^{\mathrm{NS}}$ & $0.5008^{\mathrm{NS}}$ & $0.1750^{\mathrm{NS}}$ & $0.7362^{*}$ \\
\hline & $0.7271^{*}$ & $0.4409^{\mathrm{NS}}$ & $0.5357^{\mathrm{NS}}$ & $-0.0746^{\mathrm{NS}}$ & $0.4281^{\mathrm{NS}}$ \\
\hline & $0.8292^{*}$ & $0.1390^{\mathrm{NS}}$ & $0.7781^{*}$ & $0.6828^{\mathrm{NS}}$ & 0.7207 \\
\hline
\end{tabular}

of pruning. However, the damage caused by psyllid in young leaves exhibited average with note 1 (damage between 0 and $25 \%$ of leaves), indicating that the highest population occurred after pruning was not enough to cause significant damage in the orchard. Differently, the authors reported that, in conventional cultivation system (pruning in March and November), the population of T. limbata presented peaks in April, June, August, September, October and November, even under constant applications of insecticides, with note 3 of average (between $51 \%$ and $75 \%$ damage). In the present experiment (Table 1), $82 \%$ of accesses analyzed, after pruning, showed averages notes of damage minor than 3 , showing that, in an organic system, depending on the chosen material, the infestation caused by psyllid can be smoothed.

For the management of psyllid in organic farming system of guava are recommended sprays with solution of Neem Oil to $0.5 \%(\mathrm{v} / \mathrm{v})$ in periods of high incidence of the insect [13].

The separation of the guava accesses in four groups, regarding of notes of psyllid attack symptoms (Table 1), demonstrated that the accesses used commercially, as Rica and Paluma, were more attacked by the psyllid, and the L4P14, L7P28 and L8P32B accesses showed few symptoms of attack and may indicate any resistance to insect attack. According to [14], the damage caused by the consumption of food with residues of agrochemicals have required the development of resistant cultivars and/or tolerant to pests. The development of guava cultivars resistant to psyllid and rust (Pucciniapsidii Wint.) without losses of fruit quality is a great way to be followed. In relation to psyllid, in the genetic improvement program of the Unesp/FCAV University, Jaboticabal Campus, Brazil, was developed the selection J-8501, completely resistant to this insect, however it is necessary to improve the quality of the fruit to make it commercial [14].

The cultivars sold on the market, almost in its entirety, were introduced over the years by the producers themselves. This promotes the non-recognition of improvement efforts, since, when the consumers are asked about guava cultivar, we realized that the only existing information is regarding the color of flesh, red or white [15].

It is necessary to recognize that, in the last 50 years, all research efforts were directed towards developing high-yielding cultivars, heavily dependent on large financial inputs and technologies, mainly oriented to maximizing productivity, without major concerns about the ecological aspects. Therefore, it is expected that a long way is to be taken, in order to develop productive cultivars aimed for high efficiency in the use of inputs and appropriate technologies to organic agriculture [16].

As for the psyllid correlation with meteorological data (Table 2), it was found that the damage showed better correlation when used the average data of 14 days prior assessments, with significant and positive correlation between damage of psyllid and the minimum temperature, precipitation and relative humidity registered at 1:00 p.m. The correlation between the population fluctuation of psyllid and the climatic factors has been greatly studied by various authors, but with controversial data.

In similar work [2], by Pearson correlation test showed that there is an increase in pest population density with high temperatures. Psyllid damage already showed no correlation between the meteorological factors analyzed, indicating that the damage is directly related to the presence of psyllid in the orchard, being weather conditions a secondary factor.

Dalberto et al. [9] studied the population fluctuation of psyllid in Londrina/PR/Brazil, by use of traps, and verified, by Pearson correlation test, that maximum and average temperatures affect positively the population of adults, constituting the most important factor for the occurrence of this insect. Minimum temperatures showed no significant correlation with the psyllid, but with a tendency of reduction of population in the months that have 
reached lower minimum temperatures. The authors also reported that the rainfall during the days that the trap was in the field showed no correlation with the variation of the insect population.

Pazini\& Galli [11], by registering and correlate the natural enemies inhabitants in agro ecosystem guava with key pest and with meteorological factors, found that the population fluctuation of $T$. limbata, obtained by the average number of psyllids in control, was not changed by the meteorological factors rainfall, average and minimum temperature.

Given the controversy between the results of the influence of climatic factors on the population dynamics of psyllid, the presence of budding and the phenological stage of guava trees probably have a higher importance than climatic variables in the development of this insect. A study required for better understanding the relationship of T. limbata with temperature would be your thermic requirements, to verify the real influence of temperature on insect development [17].

Generally, it was noted, by articles cited, that somehow the temperature has positive correlation with the presence of the insect. In the present work, the psyllid damages positively correlated with increased minimum temperature, rainfall and an increase in relative humidity, factors that stimulate the growth of plants. As has already been said, the insect has preference in attack new shoots, suggesting that the climate data interfere more in the host plants than in the pest.

\section{Conclusions}

Guava accesses used commercially are more susceptible to the attack of psyllid, compared to the selections, with an emphasis on the access "L4P14", "L7P28" and "L8P32B", selected by the Agronomic Institute/IAC/ Campinas/Brazil, which showed few symptoms of attack and may indicate any resistance to psyllid Triozoida sp.

The visible damages of psyllids in leaves of guava are correlated positively with the minimum temperature, rainfall and relative humidity in the period of 14 days preceding the evaluations.

\section{Acknowledgements}

We thank the Fundação de Amparo a Pesquisa do Estado de São Paulo (FAPESP), by financial assistance in the conduct of this work (Process 2012/03807-0).

\section{References}

[1] Gavioli, V.O. and Takakura, V. (2001) Goiaba oferece vantagens nutricionais e econômicas. Informativo Agropecuário Coopercitrus, 16, 18-21.

[2] Colombi, C.A. and Galli, J.C. (2009) Dinâmica populacional e evolução de dano de Triozoidalimbata (Hemiptera: Psillydae) em goiabeira, em Jaboticabal-SP. Ciência e Agrotecnologia, 33, 412-416. http://dx.doi.org/10.1590/S1413-70542009000200008

[3] Gallo, D., Nakano, O., Carvalho, R.P.L., Baptista, G.C., Berte Filho, E.B., Parra, J.R., Zucchi, R.A., Alves, S.B., Vendramin, J.D., Marchini, L.C., Lopes, J.R.E. and Omoto, C. (2002) Entomologia agrícola. FEALQ, Piracicaba.

[4] Martins, J.C. (2008) Variação sazonal de Triozoidalimbata (Hemiptera: Triozidae) e inimigos naturais em goiabeira. Ph.D. Dissertation, Universidade Federal de Viçosa, Viçosa.

[5] Harada, D.Y. (2009) Rastreabilidade e normas para a produção e cultivo orgânico de goiaba. In: Natale, W., Rozane, D.E., Souza, H.A. and Amorim, D.A., Eds., Cultura da goiaba: Do plantio a comercialização, FCAV, Jaboticabal, 285-308.

[6] Hopkins, G.W. and Memmot, J. (2003) Seasonality of a Tropical Leaf-Mining Moth: Leaf Availability versus EnemyFree Space. Ecological Entomology, 28, 687-693. http://dx.doi.org/10.1111/j.1365-2311.2003.00552.x

[7] Wallner, W.E. (1987) Factors Affecting Insect Population Dynamics: Differences between Outbreak and Non-Outbreak Species. Annual Review of Entomology, 32, 317-340.

[8] Barbosa, F.R., Souza, E.A., Siqueira, K.M.M., Moreira, W.A., Alencar, J.R. and Haji, F.N.P. (2001) Eficiência e seletividade de inseticidas no controle de psilídeo (Triozoidasp.) em goiabeira. Pesticida-Revista de Ecotoxicologia e Meio Ambiente, 11, 45-52. http://ojs.c3sl.ufpr.br/ojs/index.php/pesticidas/article/view/3134/2507

[9] Dalberto, F.M.S., Menezes Junior, A.O., Simões, H.C., Benito, N.P. and Pitwak, J. (2004) Flutuação populacional do psilídeo-da-goiabeira, Triozoidalimbata (Hemiptera: Psillydae) na região de Londrina, Paraná, PR. Semina: Ciências Agrárias, 25, 87-92. http://www.uel.br/revistas/uel/index.php/semagrarias/article/view/2219/1904

[10] Lima, J.O.G. and Gravina, G.A. (2009) Failure of Imidacloprid and Thiadoprid to Control the Guava Psyllid, Triozoi- 
dalimbata (Enderlein) (Hemiptera: Psyllidae). Ciência e Agrotecnologia, 33, 1888-1891. http://dx.doi.org/10.1590/S1413-70542009000700032

[11] Pazini, W.C. and Galli, J.C. (2011) Redução de aplicações de inseticidas através da adoção de táticas de manejo integrado de Triozoidalimbata (Enderlein, 1918) (Hemiptera: Triozidae) em goiabeira. Revista Brasileira de Fruticultura, 33, 66-72.

[12] Duarte, R.T., Galli, J.C., Pazini, W.C. and Calore, R.A. (2012) Dinâmica populacional de Triozoidalimbata, Costalimaitaferruginea e inimigos naturais em pomar orgânico e convencional de goiaba. Revista Brasileira de Fruticultura, 34, 727-733. http://dx.doi.org/10.1590/S0100-29452012000300011

[13] Guimarães, R.N.A., Freitas, G.B., Trivellato, M.D. and Pinheiro, S.S.C. (2003) Normas para certificação e cultivo orgânico da goiabeira. In: Couto, F.A.A. and Rozane, D.E., Eds., Cultura da goiabeira: Tecnologia e mercado, Universidade Federal de Viçosa, Viçosa, 333-350.

[14] Pereira, F.M. and Nachtigal, J.C. (2009) Melhoramento genético da goiabeira. In: Natale, W., Rozane, D.E., Souza, H.A. and Amorim, D.A., Eds., Cultura da goiaba: Do plantio a comercialização, FCAV, Jaboticabal, 371-398.

[15] Watanabe, H.S. (2009) Comercialização de goiaba no mercado nacional. In: Natale, W., Rozane, D.E., Souza, H.A. and Amorim, D.A., Eds., Cultura da goiaba: Do plantio a comercialização, FCAV, Jaboticabal, 133-150.

[16] Neves, M.C.P., Medeiros, C.A.B., de Almeida, D.L., De-Polli, H., da R. Rodrigues, H., Guerra, J.G.M., Nunes, M.U.C., Cardoso, M.O., dos S.F.R. Azevedo, M., de C.M.T. Vieira, R. and de O. Saminêz, T.C. (2000) Agricultura Orgânica: Instrumento para a Sustentabilidade dos Sistemas de Produção e Valoração de Produtos Agropecuários. Embrapa Agrobiologia, Seropédica.

[17] de Melo, G. (2009) Dinâmica populacional e inimigos naturais de Triozoidalimbata (Hemiptera: Triozidae) e diversidade de famílias de himenópteros parasitoides em pomar convencional e orgânico de goiaba na região de Campinas, SP. Ph.D. Dissertation, Instituto Biológico, São Paulo. 